\title{
Clinical Informatics Shared Resource
}

National Cancer Institute

\section{Source}

National Cancer Institute. Clinical Informatics Shared Resource. NCI Thesaurus. Code C39356.

The Clinical Informatics Shared Resource provides database and application support to the clinical research programs in Cancer Center including overall informatics support for clinical operations, patient registry services, development of clinical trials management applications, laboratory information management applications, privacy protection components necessary to maintain HIPAA-compliant research information services, storage, archival and other informatics services. 